\title{
VARIABILIDAD EN LOS CONTROLES DE LA TERAPIA ANTICOAGULANTE ORAL ENTRE ATENCIÓN PRIMARIA Y HOSPITAL EN ALBACETE (2009)
}

\author{
Juan Miguel Armero Simarro (1), Ma Aranzazu Romero Cebrián (2), Ma Candelaria Ayuso Raya (3), \\ María Arias Alaminos (3), Nieves Pérez López (3) y Francisco Escobar Rabadán (3).
}

(1) Centro de Salud Zona V de Albacete. Servicio de Salud de Castilla-La Mancha (SESCAM).

(2) Hospital General de Villarrobledo. Servicio de Salud de Castilla-La Mancha (SESCAM).

(3) Centro de Salud Zona IV de Albacete. Servicio de Salud de Castilla-La Mancha (SESCAM).

\section{RESUMEN}

Fundamento: Tras asumir el control de la terapia anticoagulante oral en nuestro centro de salud surgió la necesidad de detectar posibles diferencias con respecto al seguimiento hospitalario. El objetivo de nuestro trabajo fue conocer la variabilidad en los valores del Cociente Normalizado Internacional (INR) de los pacientes en tratamiento anticoagulante oral (TAO), y su posible relación con el control en centro de salud u hospital.

Métodos: Se analizaron los 6 últimos controles de 291 pacientes de un centro de salud urbano en control rutinario por TAO. A fin de analizar su variabilidad, se ha calculado la desviación estándar (DE) de los logaritmos neperianos (ln) de los valores de INR, comparando por medio del test $t$ de Student la media de las mismas entre aquellos pacientes cuyo tratamiento había sido pautado por profesionales del centro de salud y aquellos pautados en unas ocasiones por éstos y en otras en hospital. Se comparó asimismo la proporción de controles dentro de rango en cada grupo (chi2).

Resultados: Eran mujeres $153(52,6 \%)$ de los pacientes, y la media de edad era 73,8 años (DE: 11,3 ). Se han analizado un total de 1.710 controles de INR, de los que 1.412 han sido validados por médicos de familia y 298 por hematólogos, sin que existieran diferencias significativas en los valores de INR analizados por unos y otros. Los pacientes cuyo tratamiento había sido pautado siempre por profesionales del centro de salud presentaron unos valores medios de DE de los $\ln$ de los INR de los 6 controles analizados significativamente inferior $(\mathrm{p}<0,0001)$ al de aquellos que habían llevado un control mixto. Asimismo, presentaban una mayor proporción de controles dentro de rango (un $68,0 \%$ vs $38,6 \% ; \mathrm{p}<0,0001$ ).

Conclusiones: Los pacientes que son seguidos exclusivamente por los profesionales del centro de salud presentan una menor variabilidad y más adecuados controles de INR que aquellos con un control mixto centro de salud/hospital.

Palabras clave: Anticoagulantes. Cociente Normalizado Internacional. Atención Primaria de Salud.

\section{ABSTRACT}

\section{Variability in the control of Oral Anticoagulant Therapy between Primary Care and Hospital in Albacete (2009)}

Background: After taking control of oral anticoagulant therapy in our health center it became necessary to detect possible differences with regard to hospital monitoring. The aim of our study was to determine the variability in the International Normalized Ratio (INR) values of patients on oral anticoagulant therapy (OAT), and the possible relation to control in primary care or hospital.

Methods: We analyzed the last 6 controls of 291 patients in an urban health centre in routine control by OAT. In order to analyze the variability, we calculated the standard deviation (SD) of natural logarithm (ln) of INR values, comparing by Student $t$ test their mean between patients whose treatment had been scheduled in primary care and those on some occasions ruled by them and others in hospital. We compared also the proportion of controls within range in each group (chi2).

Results: $153(52.6 \%)$ patients were women, and the mean age was 73.8 years (SD: 11.3). We analyzed a total of 1710 INR controls, of which 1412 have been validated by family physicians and 298 by haematologists; there were no significant differences in INR values. Patients whose treatment had been scheduled by professionals of the health centre had mean values of the SD of the $\ln$ of the INR of 6 controls analyzed significantly lower ( $\mathrm{p}<0.0001)$ than in those who had taken a mixed control. Also, a higher proportion of controls within range $(68.0 \%$ vs $38.6 \%$, p <0.0001).

Conclusions: Patients who are followed only by professionals in the health centre have less variability and more appropriate INR control than those with a mixed control health centre / hospital.

Key words: Anticoagulants. International Normalized Ratio. Primary Health Care.

\footnotetext{
Francisco Escobar Rabadán

C/ Seminario, 4

02006 Albacete

Francisco.Escobar@uclm.es
} 


\section{INTRODUCCIÓN}

Hay numerosas evidencias, establecidas mediante ensayos clínicos bien diseñados, para utilizar el tratamiento anticoagulante oral en la prevención primaria y secundaria de una serie de situaciones clínicas muy precisas, para las que están definidos sus correspondientes rangos terapéuticos ${ }^{1-}$ ${ }^{6}$. La prueba de laboratorio utilizada como control de la terapia anticoagulante oral (TAO) es el tiempo de protrombina (TP), que ha sido estandarizada internacionalmente en forma del denominado "International Normalized Ratio" (INR) o "Cociente Normalizado Internacional", que permite comparar los resultados obtenidos por diferentes laboratorios con distintos reactivos. Para la realización de estos controles se dispone de coagulómetros portátiles $\left(\text { CoaguChek }^{\mathrm{R}}\right)^{7}$ utilizando una muestra de sangre capilar. La obtención de los resultados es inmediata, en un minuto, siendo los resultados de INR equivalentes a los obtenidos en laboratorio con muestra de sangre venosa ${ }^{8,9}$.

Tradicionalmente se ha considerado difícil el manejo de los pacientes anticoagulados, debiendo valorar siempre el riesgo individual de hemorragia ${ }^{10}$ frente al beneficio de la terapia, por lo que su control ha sido mayoritariamente hospitalario. Tanto en España como en otros muchos países, en los últimos años se ha planteado la opción de desarrollar dicho control en las consultas de Atención Primaria (AP) por varias razones: la mayor seguridad actual en la TAO, debido a la estandarización de la medición del tiempo de protrombina y a la disminución de los niveles de anticoagulación recomendados; menor frecuencia de complicaciones hemorrágicas; aumento de las indicaciones de los anticoagulantes orales; incremento del número de pacientes anticoagulados $\mathrm{y}$ de su edad media; y, fundamentalmente, por el desarrollo de la AP y la mayor capacitación de sus profesionales ${ }^{11}$.
Con estos cambios se pretende una mayor comodidad para el paciente, así como conseguir un seguimiento integral garantizando la solución de incidencias en el momento de la visita. La administración de fármacos que presentan un estrecho rango terapéutico exige la realización de controles periódicos que informen sobre el grado de anticoagulación del paciente al objeto de administrar dosis eficaces con la mínima incidencia de efectos adversos, tanto por defecto como por exceso de acción farmacológica. Por ello, el ámbito de la AP resulta el marco idóneo para poder entrevistar a los pacientes acerca del cumplimiento correcto del tratamiento, así como de cambios en la dieta y la toma de medicación o sus posibles interacciones. Permite también realizar controles en cortos períodos de tiempo con pequeños cambios en las dosis que permitan conseguir rangos correctos. La descentralización del control de la TAO supone un avance en la accesibilidad del paciente ${ }^{11}$.

En nuestro medio este control era realizado en centros hospitalarios hasta el año 2008, cuando AP asumió el cambio con incertidumbre y prudencia, con profesionales insatisfechos por la obligación de asumir una nueva competencia en la históricamente "sobrecargada" AP y que temían por una mayor saturación en sus consultas. Sin embargo esta actividad tiene un carácter eminentemente asistencial, que la ha hecho preferida a otras que se realizan en nuestro ámbito de contenido puramente burocrático. Las causas de que todavía existan pacientes controlados por el Servicio de Hematología no guardan probablemente relación con una mayor dificultad clínica de los pacientes, si no más bien con motivos organizativos o de recursos.

Tras los primeros meses de realizar esta actividad nos planteamos la necesidad de conocer cómo estaba siendo el control de los pacientes anticoagulados en nuestro centro de salud y si éste se había visto 
afectado. Nuestra hipótesis es que los pacientes cuyos controles de TAO son realizados por su médico de familia tienen cifras de INR más estables y están más frecuentemente en rango terapéutico que aquellos en cuyo control intervienen también profesionales hospitalarios.

El objetivo del trabajo es conocer la variabilidad en los valores de INR de los pacientes en TAO, y su posible relación con el nivel de atención en el que se realiza el control, centro de salud u hospital.

\section{MATERIAL Y MÉTODO}

Diseñamos un estudio de cohorte histórica, seleccionando a todos los pacientes del Centro de Salud Universitario Zona IV de Albacete que seguían controles rutinarios por TAO, de los que analizamos sus 6 últimas visitas. En aquellos que tenían realizadas menos visitas se analizaron solo las disponibles hasta el momento de realizar el estudio, en marzo y abril de 2009.

Los datos fueron obtenidos de las historias clínicas informáticas y del programa TAOCAM, aplicación informática específica, asociada al programa de consulta de atención primaria, para facilitar la gestión de la TAO. En cada control se genera una hoja de seguimiento para el paciente que incluye datos de filiación, diagnóstico que motiva la $\mathrm{TAO}$, fármaco anticoagulante y presentación, fecha de inicio de TAO, rango de INR recomendado, duración de la TAO, fecha de realización del control actual, INR de los últimos controles (todas las determinaciones fueron realizadas en sangre capilar por medio de CoaguChekR), nueva dosis total semanal (DTS), pauta diaria de TAO y fecha para el próximo control.

La información recogida fue codificada e introducida en una base de datos informática, utilizando el programa SPSS 15.0. El análisis estadístico se ha orientado a analizar la dispersión de los valores de INR en los diferentes controles realizados a cada paciente. Dado que esta variable no tiene distribución normal, se procedió a su transformación logarítmica. Se calculó la desviación estándar (DE) de los logaritmos neperianos (ln) de los valores de INR, comparando sus valores medios con el test t de Student entre los controles en que las DTS habían sido pautadas exclusivamente en el centro de salud y aquellos que se habían realizado en unas ocasiones en el centro de salud y en otras en el hospital. Para el contraste de variables continuas sin distribución normal se utilizaron tests no paramétricos, mientras que para las categóricas se utilizó el test de Chi cuadrado.

\section{RESULTADOS}

Fueron incluidos en el estudio 291 pacientes, de los que $153(52,6 \%)$ eran mujeres, con una media de edad de 73,8 años (DE: $11,3)$, y con un rango de 24 a 95 años. El diagnóstico más frecuente por el que se había indicado la TAO (152 pacientes) era la fibrilación auricular, y el rango de INR recomendado era mayoritariamente (255 pacientes) entre 2 y 3 . No existían diferencias estadísticamente significativas en relación con todas estas variables para los sujetos con control exclusivo por médico de familia o con control mixto médico de familia/hematólogo.

Se analizaron en total 1.710 controles de INR, de los que 1.412 habían sido validados por médicos de familia y 298 por hematólogos. No se encontraron diferencias significativas en los valores de INR y dosis total semanal (figura 1), ni en cuanto a complicaciones en los pacientes en función de que el control fuera realizado por uno u otro profesional (figura 2) .

Los pacientes cuyo tratamiento había sido pautado por profesionales del centro de salud presentaron unos valores medios para la desviación estándar de los ln de los INR de los 6 controles analizados significativamen- 


\section{Figura 1}

Distribución de valores de INR y dosis total semanal (DTS) para los controles de TAO pautados en centro de salud y hospital
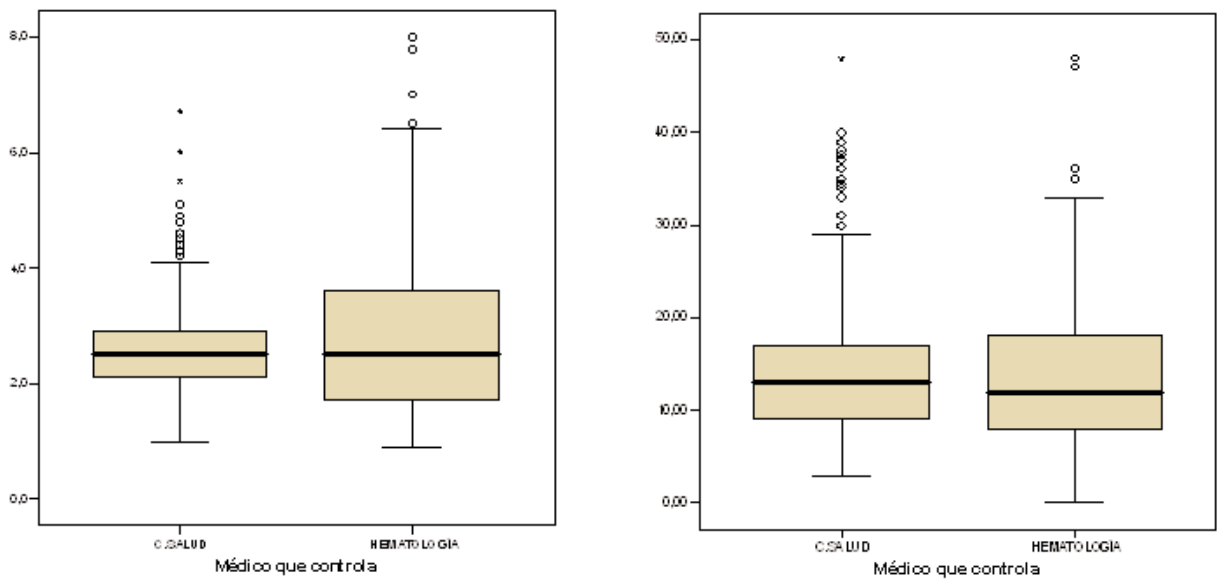

Figura 2

Complicaciones sufridas por los pacientes incluidos en el estudio en función de que hubieran tenido control exclusivo por médico de familia, o mixto médico de familia-hematólogo
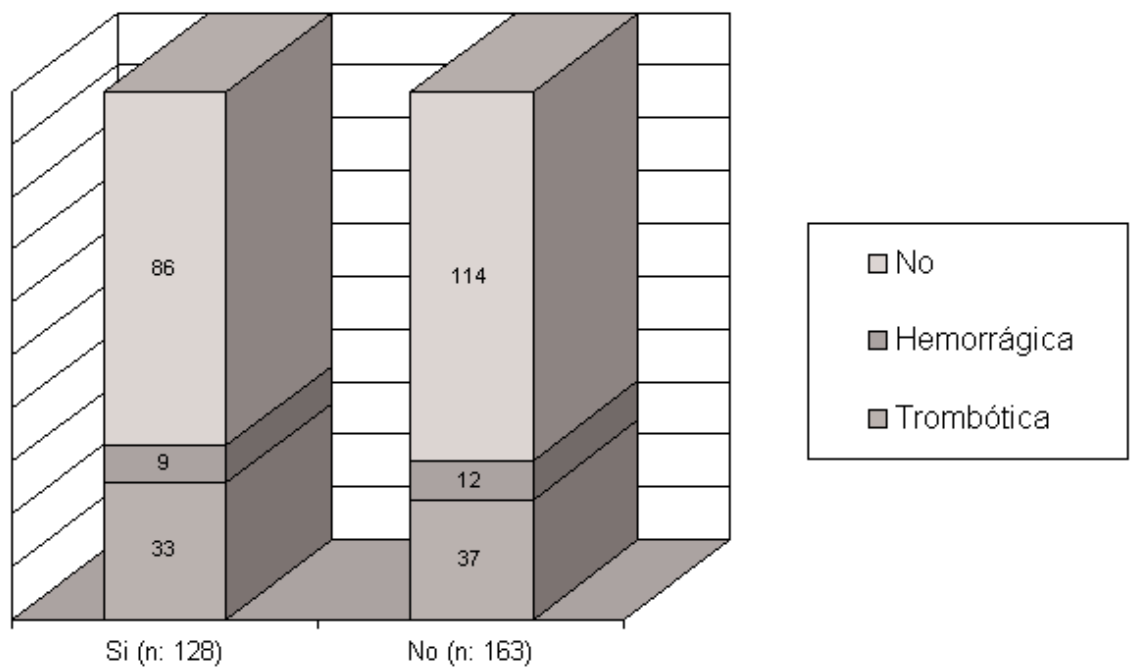

Control exclusivo Médico de Familia 


\section{Figura 3}

Distribución de la DE de los In de los INR en función de que el control fuera o no
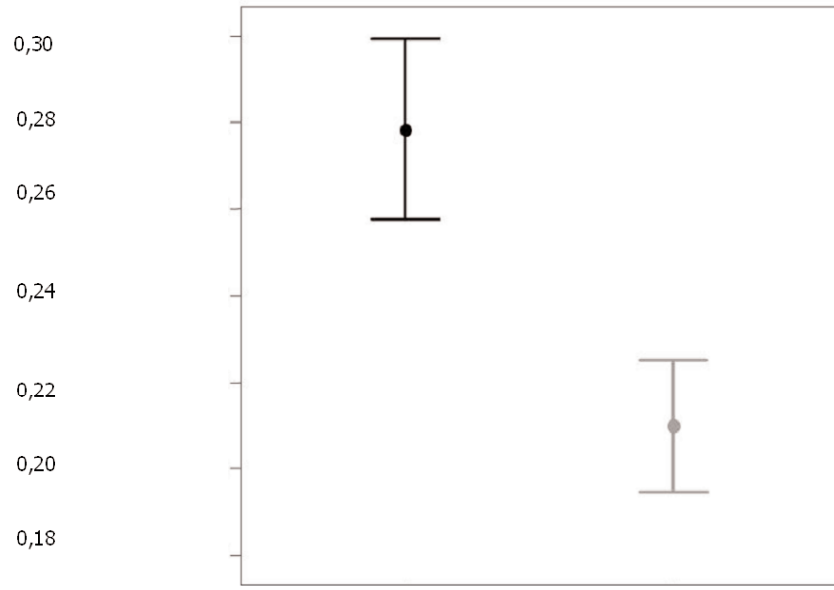

No

Si

$p<0,0001$

Control exclusivo por Médico de Familia

Figura 4

Controles de INR dentro del rango recomendado para los pacientes con control exclusivo o no por médico de familia

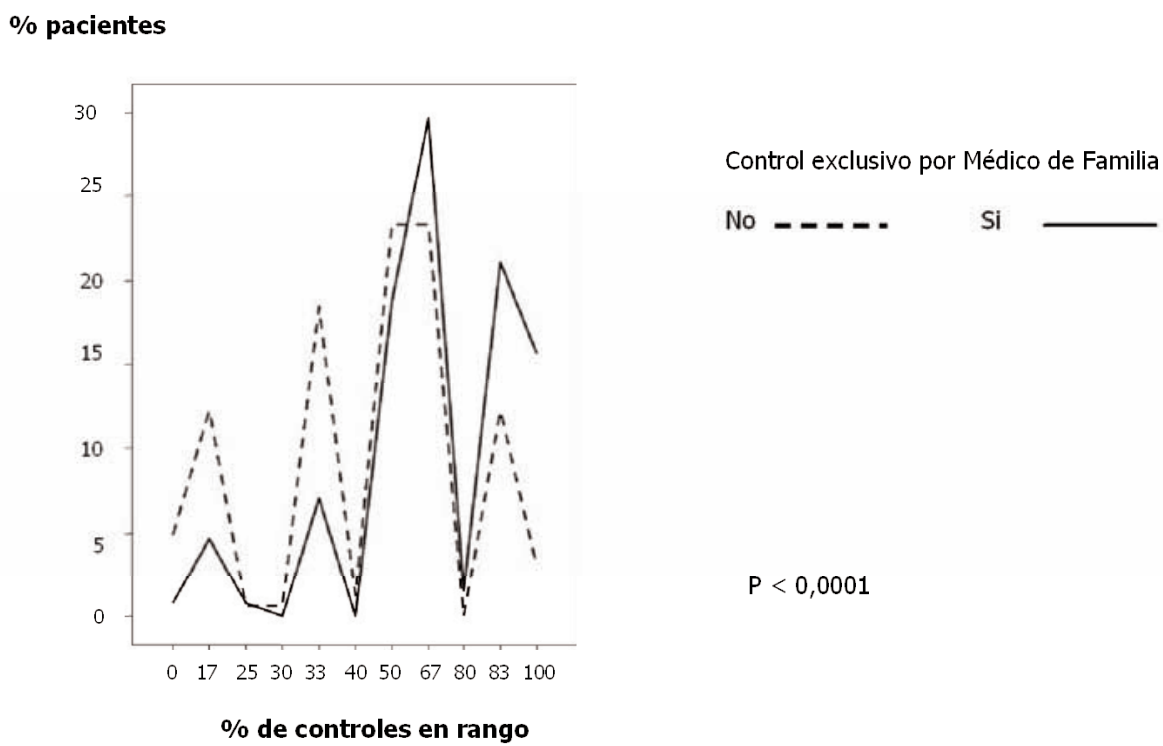


te inferior $(\mathrm{p}<0,0001)$ a los de aquellos cuyos controles se habían realizado en unas ocasiones en el centro de salud y en otras en el hospital (figura 3). Asimismo, como podemos observar en la figura 4 , presentaban una mayor proporción de controles dentro de rango: $87(68,0 \%)$ vs $63(38,6 \%)$ de los pacientes con más de la mitad de los controles en rango $(\mathrm{p}<0,0001)$.

\section{DISCUSIÓN}

Para los profesionales de nuestro centro de salud supuso un enorme reto asumir las competencias de control de pacientes en TAO. Ha resultado altamente gratificante comprobar que hemos sido capaces de realizar satisfactoriamente esta nueva tarea, con una moderada variabilidad de los valores de INR en los diferentes controles y con un elevado porcentaje de ellos dentro del rango terapéutico.

En nuestra opinión, el médico de familia cuenta con la ventaja que supone el conocimiento previo de los pacientes y la posibilidad de obtener información en el encuentro clínico con los mismos. Por el contrario los hematólogos valoran únicamente las cifras de unos pacientes con los que no tienen un contacto directo y a los que no conocen personalmente en la inmensa mayoría de los casos.

Podría objetarse que las dos poblaciones comparadas sean probablemente distintas en aspectos que podrían estar asociados con la variabilidad y el control adecuado, siendo esperable que los pacientes que requieren control mixto sean, por algún motivo, de más difícil control y/o de mayor riesgo. Sin embargo, no hemos encontrado diferencias entre estos grupos de pacientes en relación con edad, sexo, motivo por el que se indicaba la TAO, valores de INR o DTS.

Estudios similares en el ámbito de la Unión Europea demuestran la seguridad del control en una determinada población de la
TAO por $\mathrm{AP}^{12,13}$. Incluso para poblaciones seleccionadas el autocontrol del TAO mediante coagulómetros portátiles es al menos tan eficaz y seguro como el tratamiento previo habitual ${ }^{13,14}$.

Cuando concebimos nuestro estudio pensábamos contrastar el control exclusivo en centro de salud con el exclusivamente hospitalario. Sin embargo la constatación inicial de que esta última situación no se daba entre nuestros pacientes nos hizo cambiar el objetivo hacia aquellos con un control mixto. Entendemos que esto puede constituir una limitación en nuestro estudio, en tanto puede estar matizando las posibles diferencias. Otra limitación de este trabajo podría estar en el número de visitas analizadas, circunstancia condicionada por la información que proporciona el programa TAOCAM, que así mismo delimita las variables recogidas. Optamos por un diseño retrospectivo por razones de índole práctica, especialmente por la posibilidad de obtener una gran cantidad de información en un periodo relativamente corto de tiempo. Un diseño de cohorte concurrente permitiría analizar un mayor número de visitas, así como otras variables que pudieran considerarse de interés.

Quizás nuevos tratamientos ${ }^{15}$ desplacen los actuales dicumarínicos y los controles de INR queden obsoletos. Mientras llega ese momento debemos ofrecer el servicio que se haya mostrado más efectivo. En este sentido, nuestro estudio demuestra inequívocamente que los pacientes que son seguidos exclusivamente por los profesionales del centro de salud presentan una menor variabilidad y más adecuados controles de INR que aquellos con un control mixto centro de salud/hospital.

\section{BIBLIOGRAFÍA}

1. Singer DE, Albers GW, Dalen JE, Fang MC, Go AS, Halperin JL, Lip GY, Manning WJ. Antithrombotic therapy in atrial fibrillation: American College of Chest Physicians Evidence-Based Clinical Practice Guidelines (8th Edition). Chest. 2008;133(6 Suppl):546S-92S. 
2. Albers GW, Amarenco P, Easton JD, Sacco RL, Teal P. Antithrombotic and thrombolytic therapy for ischemic stroke: American College of Chest Physicians Evidence-Based Clinical Practice Guidelines (8th Edition). Chest. 2008;133(6 Suppl):630S-69S.

3. Kearon C, Kahn SR, Agnelli G, Goldhaber S, Raskob GE, Comerota AJ. Antithrombotic therapy for venous thromboembolic disease: American College of Chest Physicians Evidence-Based Clinical Practice Guidelines (8th Edition). Chest. 2008;133(6 Suppl):454S$545 \mathrm{~S}$.

4. Sobel M, Verhaeghe R. Antithrombotic therapy for peripheral artery occlusive disease: American College of Chest Physicians Evidence-Based Clinical Practice Guidelines (8th Edition). Chest. 2008;133(6 Suppl):815S-43S.

5. Becker RC, Meade TW, Berger PB, Ezekowitz M, O'Connor CM, Vorchheimer DA, Guyatt GH, Mark DB, Harrington RA. The primary and secondary prevention of coronary artery disease: American College of Chest Physicians Evidence-Based Clinical Practice Guidelines (8th Edition). Chest. 2008;133(6 Suppl):776S-814S.

6. Salem DN, O'Gara PT, Madias C, Pauker SG. Valvular and structural heart disease: American College of Chest Physicians Evidence-Based Clinical Practice Guidelines (8th Edition). Chest. 2008;133(6 Suppl):593S-629S.

7. CoaguChek. (citado el 6 de agosto de 2010). Disponible en:

www.coaguchek.com/es/index.php?target=/es/professionals/

8. Orellana MA, Martinez P, Sanchez MT, Aramendi M, Galera G. Valoración de los resultados obtenidos con el coagulómetro Coagucheck S en el control del tratamiento con anticoagulantes orales. Med Clin (Barc). 2003;121:134-6.

9. de Solà-Morales Serra O, Elorza Ricart JM. Coagulómetros portátiles: una revisión sistemática de la evidencia científica del autocontrol del tratamiento anticoagulante oral. Med Clin (Barc). 2005;124:321-5.

10. Hollowell J, Ruigomez A, Johansson S, Wallander MA, García-Rodríguez LA. The incidence of bleeding complications associated with warfarin treatment in general practice in the United Kingdom. Br J Gen Pract. 2003; 53: 312-4.

11. Nuin Villanueva MA, Arroyo Aniés M, Yurss Arruga I, Granado Hualde A, Calvo Herrado C, Elía Pitillas F, Ayerdi Navarro K. Evaluación del programa piloto de descentralización del control del tratamiento anticoagu- lante oral en el Servicio Navarro de Salud-Osasunbirea. Med Clin (Barc). 2005; 124:326-31.

12. Nilsson GH, Björholt I, Krakau I. Anticoagulant treatment of patients with chronic atrial fibrillation in primary health care in Sweden -a retrospective study of incidente and quality in a registered population. Fam Pract. 2004; 21: 612-6.

13. Fitzmaurice DA, Murray ET, Gee KM, Allan T F, Hobbs F D R. A randomised controlled trial of patient self management of oral anticoagulation treatment compared with primary care management. J Clin Pathol. 2002;55:845-9.

14. Connock M, Stevens C, Fry-Smith A, Jowett S, Fitzmaurice D, More D, Song F. Clinical effectiveness and cost-effectiveness of different models of managing long-term oral anticoagulation therapy: a systematic review and economic modelling. Health Technol Assess. 2007;11:1-66.

15. Wallentin L, Yusuf S, Ezekowitz MD, Alings M, Flather M, Franzosi MG, Pais P, Dans A, Eikelboom J, Oldgren J, Pogue J, Reilly PA, Yang S, Connolly SJ, on behalf of the RE-LY investigators. Efficacy and safety of davigatran compared with warfarin at different levels of international normalised ratio control for stroke prevention in atrial fibrillation: an analysis of the RE-LY trial. Lancet. 2010;376:975-83. 